First publ. in: Journal of Low Temperature Physics, 142, 1/2, pp. 83-89

\title{
Hydrogen Adsorption Isotherms: Modelling of Experimental Data
}

\author{
Stefan Tibus, Jürgen Klier, and Paul Leiderer \\ Department of Physics, University of Konstanz, 78457 Konstanz, Germany \\ E-mail: Stefan.Tibus@uni-konstanz.de
}

(Received August 10, 2005; revised October 26, 2005)

\begin{abstract}
Adsorption isotherm measurements have shown deviations from classical theories in the past, leading to the inclusion of more complex effects into adsorption theory. We demonstrate here that in the case of hydrogen adsorption above the triple point measured by surface plasmon spectroscopy the main deviation is related to the measurement method itself. Modelling of the experimental data shows good agreement with the classical theoretical expectations, without the need of additional contributions.
\end{abstract}

KEY WORDS: adsorption isotherm; hydrogen; surface plasmon spectroscopy.

\section{INTRODUCTION}

The wetting of solid substrates in a gaseous environment is an omnipresent phenomenon. Gas molecules are attracted to the substrate by molecular forces and cover the surface with a thin film whose thickness is determined by the saturation of the gas. The theories by Frenkel, Halsey, and Hill $(\mathrm{FHH})^{1-5}$ as well as by Dzyaloshinskii et al. (DLP) ${ }^{6,7}$ are based solely on the van der Waals force, being the most important attractive force involved. These have been accepted to describe adsorption isotherms accurately for a long time.

In recent years there have been attempts to account for possible contributions to the adsorption mechanism in order to explain experimentally observed deviations from the ideal FHH/DLP-behavior. These may for example be related to the surface roughness of the substrate ${ }^{8}$ and thermal fluctuations. ${ }^{9,10}$ On the other hand, we have recently presented ${ }^{11}$ experimental data using surface plasmon spectroscopy as a probe exhibiting a temperature dependence which cannot be understood by application of these theories. As we show below, however, the observed discrepancy 
(a)

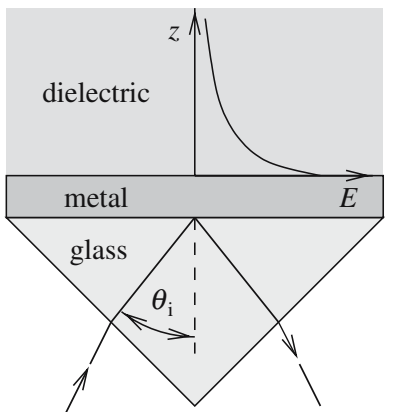

(b)

\section{hydrogen vapor}

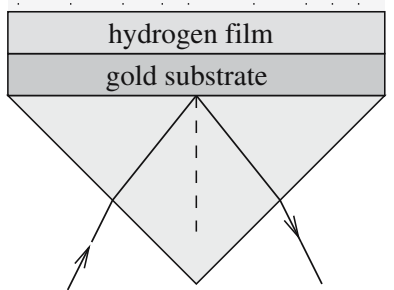

Fig. 1. Schematic of surface plasmon spectroscopy measurement. (a) Kretschmann-Raether geometry and evanescent field extending into the dielectric. (b) Layer structure as used in the experiments and for the calculations, consisting of BK7-glass prism, $47 \mathrm{~nm}$ gold substrate, hydrogen film and hydrogen vapor.

is mainly due to the measurement method, and can be resolved, when the contribution of gas atoms to the surface plasmon signal is taken into account.

\section{MEASUREMENT METHOD}

Our experiments are carried out using surface plasmon (SP) spectroscopy for measuring the adsorbed hydrogen film thickness on a gold substrate. SP spectroscopy is a well established method for the analysis of metal surfaces, ${ }^{12}$ it has been used as a sensitive technique in hydrogen wetting investigations for a long time. ${ }^{10,11,13-17}$ The method is based on the sensitivity of the SP resonance in a metallic film onto the dielectric properties in the space above it. ${ }^{18}$

We have used a geometry as presented in Fig. 1a to resonantly excite SPs by attenuated total reflection. The incident angle, $\theta_{\mathrm{i}}$, under which the SPs are excited $\left(\theta_{\mathrm{i}}=\theta_{\mathrm{r}}\right)$ is observed as a minimum of the reflected light intensity. As the electromagnetic field of the SPs decays exponentially into the space above the metal film, ${ }^{18}$ the resonance is sensitive to a coverage of the surface by a dielectric material within the decay length of some $100 \mathrm{~nm} .{ }^{15}$ The observed change of the resonance angle, $\theta_{\mathrm{r}}$, is recorded as a measure for the coverage.

In most cases of coating materials the gas density above the surface is small enough to be neglected and it is possible to derive a linear relation between resonance angle shift and small coating thicknesses. ${ }^{19}$ This has also been assumed to be the case in hydrogen adsorption experiments in the past. ${ }^{10,11,13-17}$ To verify this assumption we have 

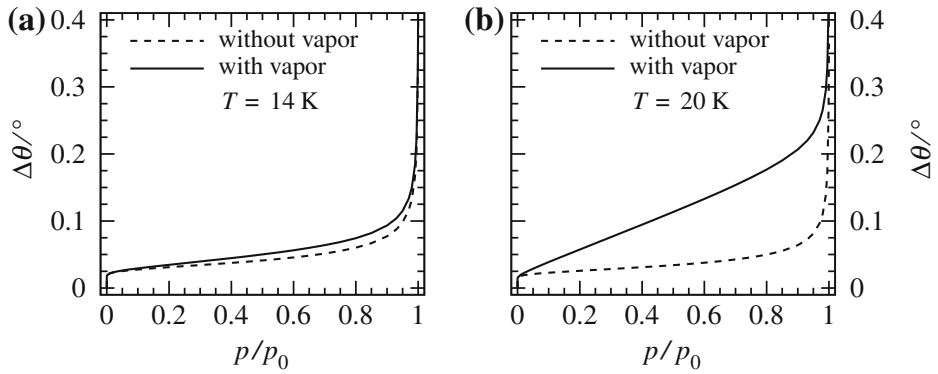

Fig. 2. Calculated surface plasmon resonance shifts (using the FHH/DLP-theory) for hydrogen adsorption isotherms without and with consideration of the vapor above the film at two different temperatures. (a) $T=14 \mathrm{~K}$, (b) $T=20 \mathrm{~K}$. The increased vapor density at the higher temperature leads to a strong increase of the resonance shift beyond the contribution by the growing liquid film. The calculations are for the geometry presented in Fig. 1 using a Hamaker constant $\Delta C_{3}=6000 \mathrm{~K} \AA^{3}$.

now performed calculations including the hydrogen vapor, modelling the system as shown in Fig. 1b. The dielectric constant of liquid hydrogen has been interpolated from literature values. ${ }^{20}$ The dielectric constants of the vapor and non-saturated gas have been calculated according to the Clausius-Mosotti law, based on density values taken from the literature. ${ }^{21}$ For the adsorbed film thickness we have assumed an ideal FHH/DLPbehavior, i.e. $d=-\Delta C_{3} /\left(k_{\mathrm{B}} T \ln p / p_{0}\right)$. Attraction of the hydrogen molecules by the glass substrate may be neglected due to the large separation by the gold film and the adsorbed films considered here are thin enough to stay in the non-retarded regime. ${ }^{22}$ Figure 2 presents the results for temperatures of 14 and $20 \mathrm{~K}$ and reveals a well noticeable additional resonance shift due to the vapor. While this contribution is on the order of $20 \%$ at $14 \mathrm{~K}$, just above the triple point $\left(T_{\mathrm{t}}=13.8 \mathrm{~K}\right)$, it grows with temperature and eventually completely overshadows the signal due to the liquid film (see Fig. $2 \mathrm{~b}, T=20 \mathrm{~K}$ ).

\section{EXPERIMENTS}

In order to compare the above calculations with experiments a new series of adsorption isotherms has been taken. To minimize dependencies on substrate properties the experiments have been carried out using a freshly prepared substrate, a thermally evaporated $47 \mathrm{~nm}$ thick gold film on a BK7-glass prism. The isotherms have been taken as in earlier experiments ${ }^{11}$ using a continuous flow of hydrogen, low enough to ensure quasiequilibrium conditions. The temperature range covered is $14-20 \mathrm{~K}$ in steps 


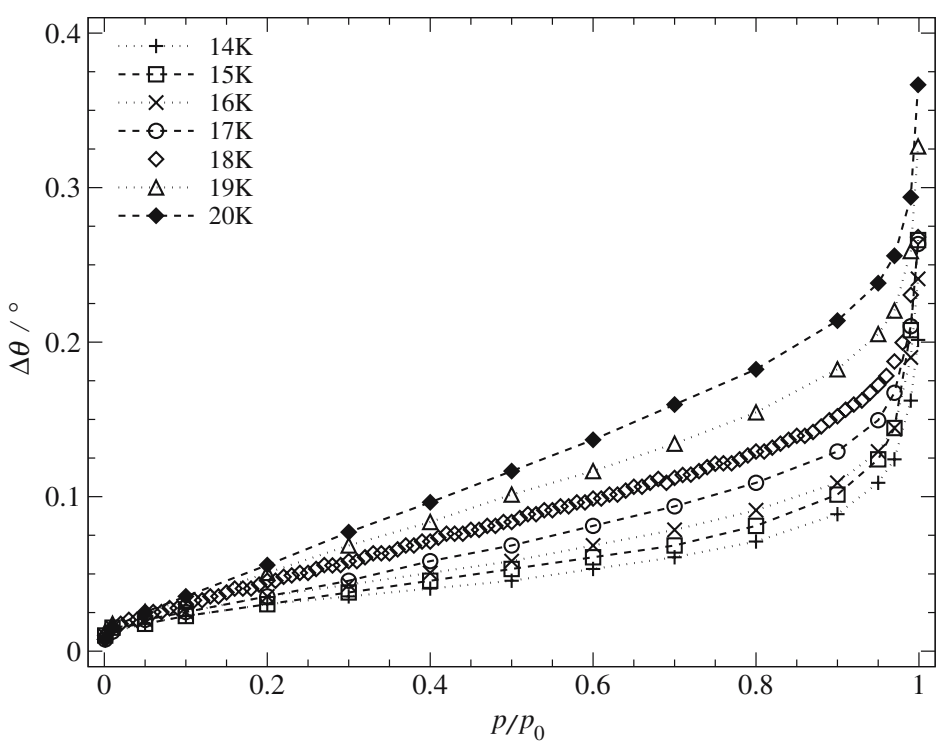

Fig. 3. Measured adsorption data in the temperature range from 14 to $20 \mathrm{~K}$. The systematic increase of the plasmon resonance shift with temperature is well pronounced. For readability the full dataset is shown for $18 \mathrm{~K}$ only. For the other temperatures selected values of $p / p_{0}$ are shown, connected by lines as a guide to the eye. The relative measurement uncertainty is about the size of the symbols (corresponding to $\approx 1 \AA$ liquid hydrogen film).

of $1 \mathrm{~K}$. As Fig. 3 shows, the systematic increase of the SP resonance shift is well observable. In addition to these measurements also desorption isotherms have been taken, which were found to overlay very well with the adsorption data, indicating that thermodynamic equilibrium conditions were fulfilled.

In Fig. 4 we present a direct comparison of the experimental and calculated data. In addition to the relative uncertainty of the measurement, which corresponds to $\approx 1 \AA$ of liquid hydrogen film, a background uncertainty of $\approx 3 \AA$ has to be considered, accounting for possible coverage of the substrate surface at the beginning of the measurement. Within this range the data points have been shifted to match the calculations at $p / p_{0}=0.3$. The experimental data then agree nicely over the whole range with the calculations for a Hamaker constant of $\Delta C_{3}=6000 \mathrm{~K} \AA^{3}$, the value of best fit at $14 \mathrm{~K}$. Only at the higher temperatures there might be a slight systematic deviation of the data towards higher resonance shifts, which in terms of the liquid film thickness corresponds to an increase of $\leq 2 \AA$. 


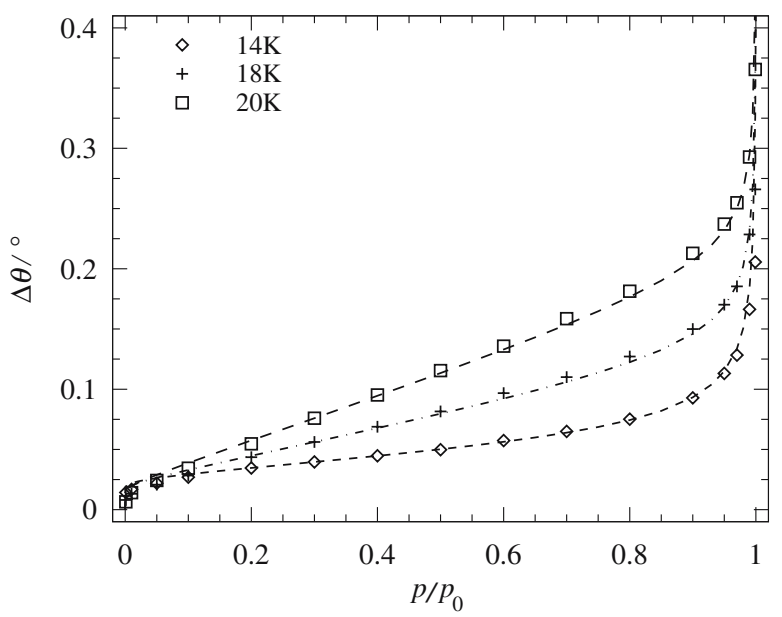

Fig. 4. Comparison of the data from Fig. 3 (symbols) to calculations (lines) at three temperatures. As in Fig. 2 a Hamaker constant of $\Delta C_{3}=6000 \mathrm{~K} \AA^{3}$ is used. For readability only a few points of the measured data are shown. The relative measurement uncertainty is about the size of the symbols.

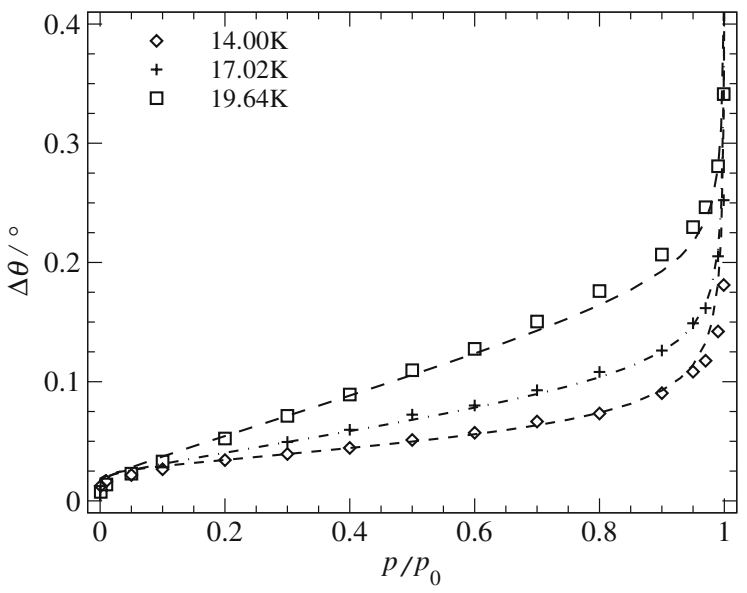

Fig. 5. Comparison of older data ${ }^{11}$ (symbols) to the new calculations (lines). For readability only a few points of the measured data are shown. The relative measurement uncertainty is about the size of the symbols.

Applying the same procedure to our previously published data ${ }^{11}$ results in the same good agreement, see Fig. 5. 


\section{CONCLUSIONS}

The comparisons in Figs. 4 and 5 show that the discrepancy between the classical FHH/DLP-theory and experimental data, as reported, ${ }^{11}$ can be removed to a large extent by taking the contribution of the vapor to the SP signal into account. The size of the effect due to the vapor is density and thus temperature dependent and can even be larger than the resonance shift by the liquid film alone. The good agreement displayed in Figs. 4 and 5 leaves room only for minor contributions by, e.g., thermal fluctuations and substrate roughness to the film thickness of the adsorbate. Especially regarding the thermal fluctuations this in contrast to published work. ${ }^{10}$

Furthermore, the analysis of our experimental data suggests a Hamaker constant $\Delta C_{3} \approx 6000 \mathrm{~K} \AA^{3}$ for molecular hydrogen on gold, based on the best fit at $14 \mathrm{~K}$. (One has to note that, as in most other experiments with hydrogen, our substrates were not prepared under UHV conditions, hence there might be a contribution from a thin adsorbed water layer.) However, the measurement uncertainties allow for $\Delta C_{3}=6000_{-2000}^{+4000} \mathrm{~K} \AA^{3}$, which is in agreement with the theoretically expected value of $\Delta C_{3}=8000 \mathrm{~K} \AA^{3} .^{22}$

\section{ACKNOWLEDGMENTS}

This work was supported by the DFG-Schwerpunkt 'Wetting and Structure Formation at Interfaces' under grant Le 315/20.

\section{REFERENCES}

1. J. Frenkel, Kinetic Theory of Liquids Clarendon, Oxford (1946).

2. G. Halsey, J. Chem. Phys. 16, 931 (1948).

3. T. Hill, J. Chem. Phys. 17, 520 (1949).

4. T. Hill, J. Chem. Phys. 17, 590 (1949).

5. T. Hill, J. Chem. Phys. 17, 668 (1949).

6. I. Dzyaloshinskii, E. Lifshitz, and L. Pitaevskii, Sov. Phys. JETP 37, 161 (1960).

7. I. Dzyaloshinskii, E. Lifshitz, and L. Pitaevskii, Adv. Phys. 10, 165 (1961).

8. J. Klier, M. Zech, A. Fubel, P. Leiderer, and V. Shikin, J. Low Temp. Phys. 138, 355 (2005).

9. K. Mecke and J. Krim, Phys. Rev. B 53, 2073 (1996).

10. J. Vorberg, S. Herminghaus, and K. Mecke, Phys. Rev. Lett. 87, 196105 (2001).

11. S. Tibus, J. Klier, and P. Leiderer, J. Low Temp. Phys. 139, 531 (2005).

12. S. Herminghaus and P. Leiderer, Appl. Phys. Lett. 58, 352 (1991).

13. S. Herminghaus, J. Vorberg, H. Gau, R. Conradt, D. Reinelt, H. Ulmer, P. Leiderer, and M. Przyrembel, Ann. Physik 6, 425 (1997).

14. U. Albrecht, P. Evers, and P. Leiderer, Surf. Sci. 283, 419 (1993).

15. R. Conradt, Ph.D. thesis, University of Konstanz (1996).

16. J. Vorberg, Ph.D. thesis, University of Potsdam (1997).

17. M. Sohaili, Ph.D. thesis, University of Konstanz (2004). 
18. O. Marti, H. Bielefeldt, B. Hecht, S. Herminghaus, P. Leiderer, and J. Mlynek, Optics Comm. 96, 225 (1993).

19. H. Raether, Surface Plasmons, vol. 111 of Springer Tracts in Modern Physics, Springer, Berlin (1988).

20. Landolt-Börnstein, Numerical Data and Functional Relationships in Science and Technology, Springer, Berlin (1991), new Series, Group IV, Vol. 6.

21. Landolt-Börnstein, Zahlenwerte und Funktionen, Springer, Berlin (1960). sechste Auflage, Band II, Teil 2.

22. E. Cheng and M. Cole, Phys. Rev. B 38, 987 (1988). 